\title{
BH3 profiling discriminates on-target small molecule BH3 mimetics from putative mimetics
}

\author{
Mariana Villalobos-Ortiz ${ }^{1}$ Jeremy Ryan (i) $^{1} \cdot$ Thelma N. Mashaka ${ }^{1} \cdot$ Joseph T. Opferman $^{2} \cdot$ Anthony Letai $^{1,3,4,5}$
}

Received: 14 December 2018 / Revised: 20 June 2019 / Accepted: 24 June 2019 / Published online: 22 July 2019

(c) The Author(s), under exclusive licence to ADMC Associazione Differenziamento e Morte Cellulare 2019

\begin{abstract}
Inhibition of the anti-apoptotic machinery of cancer cells is a promising therapeutic approach that has driven the development of an important class of compounds termed "BH3 mimetics". These novel small molecules mimic BH3-only proteins by antagonizing the pro-survival function of anti-apoptotic proteins, thereby inducing apoptosis in cancer cells. To qualify as an authentic BH3 mimetic, a compound must function directly on the mitochondria of a cell of known antiapoptotic dependence, must directly and selectively inhibit the anti-apoptotic protein with high-affinity binding, and must induce mitochondrial outer membrane permeabilization (MOMP) and apoptosis in a BAX/BAK-dependent manner. While many BH3 mimetics have entered clinical trials, the lack of a reliable validation assay to directly test the mitochondrial activity of new $\mathrm{BH} 3$ mimetic candidates has resulted in many misleading reports of agents touted as $\mathrm{BH} 3$ mimetics despite their off-target mechanisms of action. BH3 profiling probes the activity of a compound at the mitochondrial level by measuring cytochrome $\mathrm{c}$ release as a surrogate marker for MOMP. We propose a comprehensive biochemical toolkit consisting of BH3 profiling in parallel with high-throughput Annexin V/Hoechst viability testing to validate BH3 mimetic candidates. We tested our toolkit on eighteen different putative BH3 mimetics using a set of standardized cell lines of known anti-apoptotic dependence. Included in this set of cell lines is an apoptosis refractory BAX/BAK DKO control line to detect compounds that function independently of the BCL-2 family. Taken together, this rapid, efficient means of testing will prove advantageous as the demand for $\mathrm{BH} 3$ mimetics increases, particularly in the quest to identify and develop more potent MCL-1 inhibitors for use in the clinic. We strongly urge researchers utilizing BH3 mimetics in their work to use the potent and selective compounds identified with this validation toolkit instead of those lacking such potency and selectivity.
\end{abstract}

These authors contributed equally: Mariana Villalobos-Ortiz, Jeremy Ryan

\section{Edited by G. Melino}

Anthony Letai

anthony_letai@dfci.harvard.edu

1 Department of Medical Oncology, Dana-Farber Cancer Institute, 02215 Boston, MA, USA

2 Department of Cell and Molecular Biology, St. Jude Children's Research Hospital, Memphis, TN, USA

3 Department of Medicine, Brigham and Women's Hospital, Boston, MA, USA

4 Harvard Medical School, Boston, MA, USA

5 Ludwig Center at Harvard, Boston, MA, USA

\section{Introduction}

Many cancer cells must block apoptotic signaling in order to survive [1]. The mitochondrial pathway of apoptosis is regulated by more than a dozen proteins of the BCL-2 family, which is divided into three groups: the pro-apoptotic multi-domain, the anti-apoptotic multi-domain, and the BH3-only pro-apoptotic proteins. Their interactions at the mitochondrial outer membrane ultimately decide if the pore-forming multi-domain pro-apoptotic proteins BAX or BAK will undergo a conformational change, oligomerize, and form pores in the outer mitochondrial membrane [2]. Mitochondrial outer membrane permeabilization (MOMP) permits the release of intermembrane space proteins, including cytochrome $\mathrm{c}$, followed by caspase activation and apoptosis. Anti-apoptotic proteins block apoptosis by binding the monomeric pro-apoptotic proteins, preventing homo- and hetero-typic interactions among pro-apoptotic proteins that are necessary for MOMP. Anti-apoptotic 
proteins possess a hydrophobic groove that binds the hydrophobic face of the amphipathic, alpha-helical BH3 domain that is present in every BCL-2 family protein.

BH3 mimetics are a class of compounds that compete for this hydrophobic groove to antagonize the function of antiapoptotic proteins. BH3 mimetics have entered the clinic and even gained regulatory approval for use in cancer. Thus, there is justifiable enthusiasm for creating novel $\mathrm{BH} 3$ mimetics. However, many putative $\mathrm{BH} 3$ mimetics are reported in the literature, and methods for validating these compounds are inconsistent so that the relative quality of different agents is sometimes uncertain. Inhibiting the antiapoptotic members of the BCL-2 family proved to be challenging because the binding pocket is quite large, shallow, and hydrophobic. Typical small molecules cannot fully occupy this space and have struggled to be both potent and specific in their binding. The search for compounds that mimic the $\mathrm{BH} 3$ domain is usually guided by binding assays such as fluorescence polarization or surface plasmon resonance using tagged and often truncated versions of the multi-domain anti-apoptotic proteins. While these methods ignore other interactions, such as those of the mitochondrial membrane and its occupants, they have been useful in identifying, constructing, and validating early compounds.

Confusion regarding the method of action of many early inhibitors led to questions of how to better define a BCL-2 family inhibitor [3]. Binding assays alone could not predict or discriminate between on-target direct interactions, indirect cellular effects stemming from upstream signaling, or predict deleterious off-target effects. The ideal $\mathrm{BH} 3$ mimetic should operate directly on mitochondria, would selectively induce MOMP in mitochondria dependent on the specific anti-apoptotic protein target(s), and would require BAX and BAK for MOMP. Moreover, the cellular toxicity should track closely with the potency in inducing MOMP by direct mitochondrial exposure.

To perform testing of $\mathrm{BH} 3$ mimetics, we have assembled a toolkit of cell lines and BH3 peptides. Four cell lines with enforced expression of human BCL-2, BCL-XL, MCL-1, or BFL-1/A1 and one additional line that has homozygous deletion of both BAX and BAK have been engineered on a common murine B-ALL background driven by BCR-ABL with homozygous deletion of p16ARF and murine MCL-1 [4]. These five lines encompass the most common antiapoptotic dependencies, including apoptosis refractory BAX/BAK DKO cells, that serve as an important control for the detection of mitochondrial or cellular effects that are independent of the BCL-2 family and therefore undesirable in BH3 mimetics. Because their mitochondrial phenotypes are stable and predictable, they are excellent standards to compare the behavior of new BH3 mimetics. In addition, we have assembled a panel of synthetic $\mathrm{BH} 3$ peptides [5-7] of known selectivity of interaction with the anti-apoptotic proteins listed above, which can function as important positive and negative controls for credentialing our mitochondrial testing of $\mathrm{BH} 3$ mimetics. We perform this via a BH3 profiling method we call iBH3 [8], a method by which we expose mitochondria to standardized concentrations of BH3 peptides and measure MOMP via release of cytochrome c via flow cytometry.

We have tested eighteen different putative BH3 mimetics using our toolkit. We find that several possess the properties expected of bona fide, selective, and potent BH3 mimetics, but that several demonstrate important deficiencies, calling into serious question their mechanism of action. Our simple, scalable method can improve the efficiency of testing potency and validating the method of action for future BH3 mimetics as the demand for this new class of therapeutics grows.

\section{Materials and methods}

\section{Cell culture}

Murine cells bearing human anti-apoptotic proteins were created and characterized for anti-apoptotic dependence previously [4]. They were maintained in RPMI with $10 \%$ FBS, L-glutamine, NEAA, HEPES, pen/strep, and $8 \mathrm{uL}$ pure 2-mercaptoethanol per liter of media. Cells were maintained at $37{ }^{\circ} \mathrm{C}$ and $5 \% \mathrm{CO}_{2}$ at $100 \%$ humidity. Cell lines were tested for mycoplasma using a Lookout PCR mycoplasma testing kit (Sigma-Aldrich) and found to be negative.

\section{Compounds}

ABT-737, Sabutoclax, BDA-366, and UMI-77 (SellekChem, Houston, TX, USA), ABT-263 (navitoclax), WEHI-539, A-1210477, and TW-37 (ApexBio, Boston, MA USA), ABT-199 (venetoclax) and maritoclax (Medchem Express, Monmouth Junction, NJ USA), S63845 (Active Biochem, Kowloon, Hong Kong), A-1331852, A-1155463, and AMG176 (Chemietek, Indianapolis, Indiana USA), Gossypol (Sigma-Aldrich, St. Louis, MO USA), and obatoclax (LC Labs, Woburn, MA USA) were obtained as solid stocks, dissolved in DMSO, and stored at $-20^{\circ} \mathrm{C}$. AZD4320 and AZD5991 were obtained from Astra Zeneca. The Annexin V HIS plasmid was a gift of Seamus Martin, and Annexin V-Alexa647 was produced as described [9].

\section{iBH3 profiling}

$\mathrm{BH} 3$ profiling with the $\mathrm{BBH} 3$ method was performed as described [8]. Cells were suspended at $2 \times 10^{6}$ cells $/ \mathrm{mL}$ in MEB2P25 buffer (150 mM mannitol, $150 \mathrm{mM} \mathrm{KCl,} 10 \mathrm{mM}$ 
HEPES, $5 \mathrm{mM}$ succinate, $1 \mu \mathrm{M}$ EDTA, $1 \mu \mathrm{M}$ EGTA, $0.1 \%$ protease free BSA, $0.25 \% \mathrm{w} / \mathrm{v}$ polaxamer 188 , final $\mathrm{pH} 7.4$ ) and added to plates containing peptides at $2 \times$ their final concentration in MEB2P25 buffer with $0.002 \% \mathrm{w} / \mathrm{v}$ digitonin. Cells were exposed at $25^{\circ} \mathrm{C}$ for $60 \mathrm{~min}$ before fixation, neutralization, and staining. Cells were stained with Hoechst 33342 (Thermo Fisher, Waltham, MA USA) and anti-cytochrome c (BioLegend, San Diego, CA USA). Fluorescent cytometry to measure cytochrome c intensity was performed using an Intellicyt iQue Screener Plus (Intellicyt, Albuquerque, NM USA). The raw data were converted to the cytochrome c loss parameter as described below in Data Reduction. Additional protocol details and comparison of $\mathrm{BH} 3$ profiling techniques can be found online at https://letailab.dana-farber.org/bh3-profiling.html. Fluorescence from obatoclax and ABT-199 were avoided by using cytometer channels that did not show significant background from these drugs.

\section{Viability testing}

Cells were plated at $0.5 \times 10^{6}$ cells $/ \mathrm{mL}$ in culture medium in 96-well plates with dilutions of each compound, total volume $0.1 \mathrm{~mL}$, and incubated for $24 \mathrm{~h}$. $10 \mathrm{uL}$ of $10 \mathrm{X}$ Annexin $\mathrm{V}$ binding buffer (100 mM HEPES, $40 \mathrm{mM} \mathrm{KCl}$, 1.4 M NaCl, 7,5 mM MgCl2, $25 \mathrm{mM} \mathrm{CaCl} 2 \mathrm{pH}$ 7.4) with Annexin V-Alexa647 [9] and $20 \mathrm{ug} / \mathrm{mL}$ Hoechst 33342 was added and incubated at RT for $15 \mathrm{~min}$. Cells were fixed by adding $30 \mathrm{uL}$ of $4 \mathrm{X}$ Annexin V Fix buffer (4\% formaldehyde, $0.5 \%$ glutaraldehyde in $1 \mathrm{X}$ Annexin $\mathrm{V}$ binding buffer) for $10 \mathrm{~min}$ at RT, neutralized using N2 buffer $(1.7 \mathrm{M}$ Tris, 1.25 M glycine $\mathrm{pH} 9.1$ ), and analyzed for Annexin V positivity on the iQue Screener Plus. Populations were scored as Live (Hoechst + Annexin $\mathrm{V}-$ ), early apoptotic (Hoechst + Annexin $\mathrm{V}+$ ), and late apoptotic (Hoechst low Annexin $\mathrm{V}+$ ). Because Hoechst 33342 stains all cells, live cells show as $2 \mathrm{~N}+4 \mathrm{~N}$ populations that can persist into early apoptosis, and late apoptotic cells show a weaker staining due to nuclear degradation.

\section{Data reduction}

Our calculations were based on the distributions of cytochrome $\mathrm{c}$ intensity in test and control samples. For each distribution, we calculated a median fluorescence intensity (MFI). For each data point, \% cytochrome c loss was calculated using the equation described in ref. [7] and listed below.

$$
\begin{aligned}
& \% \text { Cytochrome C Loss }= \\
& \qquad 1-\left[\left(\mathrm{MFI}_{\text {Sample }}-\mathrm{MFI}_{\mathrm{FMO}}\right) /\left(\mathrm{MFI}_{\mathrm{Buffer} \text { Control }}-\mathrm{MFI}_{\mathrm{FMO}}\right)\right.
\end{aligned}
$$

"Sample" is the treatment well being analyzed, "FMO" is a control with no cytochrome c antibody that defines the minimum possible cytochrome c signal, and the "Buffer control" is a well with all assay components but no peptide or drug, which defines complete cytochrome c retention. Sigmoidal dose-response curves of \% cytochrome c loss versus drug concentration were then fit using a non-parametric fit with variable Hill slope and lower and upper bounds set to 0 and 100, respectively. Data were thus normalized and graphed using PRISM (Graphpad Software, San Diego, CA USA).

\section{Results}

To make a useful comparison, we first limited several factors including exposure time, compound concentration, and media composition for both mitochondrial and cellular assays. An effective BH3 mimetic should cause death quickly, often within a few hours, so we chose $24 \mathrm{~h}$ as our cutoff for cell viability measurements by Annexin V. While we understand that serum binding may reduce the effective concentration to which the cells are exposed, serum binding will also be present in vivo, and we controlled for this by testing all compounds under identical serum concentration. Maximum drug concentrations were chosen to ensure all compounds were soluble in assay buffers and culture media. Ranges of concentrations were chosen to ensure a good dynamic range that ideally went from no response to full response. Note that $1 \mu \mathrm{M}$ is within this range, roughly the serum concentration achieved by the one FDA-approved BH3 mimetic, venetoclax [10], when used as recommended. For each compound tested, the above conditions were applied equally to all five cell lines in $\mathrm{iBH} 3$ profiling to show direct mitochondrial activity, and in cell death assays using Annexin $\mathrm{V}$ to show activity against intact cells. Both Annexin $\mathrm{V}$ and iBH3 were performed using assays terminating in fixation and performed with liquid handlers to demonstrate the utility of the assay in an automated format.

We have previously described the creation of a set of murine leukemia cell lines, each with a defined dependence on a different anti-apoptotic protein [4], including BCL-2, BCL-XL, MCL-1, and BFL-1. In addition, one control cell line lacked the pro-apoptotic effector proteins BAX and BAK. We used cellular toxicity, as measured by Annexin $\mathrm{V}$, and mitochondrial toxicity, as measured by cytochrome $\mathrm{c}$ release on direct mitochondrial exposure, as measures of the properties of putative $\mathrm{BH} 3$ mimetic compounds. We first validated the anti-apoptotic dependence of the cell lines with the BH3 peptides. Figure 1a shows the interaction between BH3 peptides and the anti-apoptotic proteins, as previously determined by fluorescence polarization or surface plasmon resonance [5-7]. Figure 1b shows the MOMP response to the $\mathrm{BH} 3$ peptide panel. In each case, the MOMP response validated the anti-apoptotic dependence of the cell line and mitochondria. Note that the cell line lacking BAX 


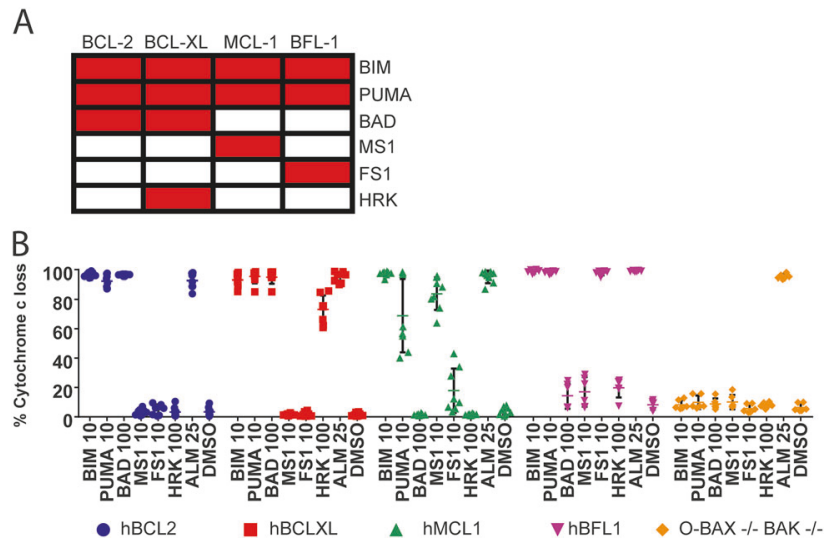

Fig. 1 a The binding pattern of anti-apoptotic proteins at the top vs. the synthetic BH3 peptides at right. A red box indicates high-affinity binding, with $\mathrm{Kd}$ values of $<100 \mathrm{nM}$ as measured by fluorescence polarization (BIM, PUMA, BAD, HRK) [5] or surface plasmon resonance (MS1, FS1) [6, 7]. b Control BH3 profiles for each cell line showing that cell line dependencies match with the interaction specificities shown in the binding assays. ALM is alamethicin, a BAX/BAK independent pore-forming peptide used as a release control. $N=6-8$ and bars show mean and standard deviation

and $\mathrm{BAK}$ was completely resistant to $\mathrm{BH} 3$ peptide-induced MOMP, as expected.

We next examined six putative $\mathrm{BH} 3$ mimetics, including gossypol [11], obatoclax [12], subatoclax [13], maritoclax [14], TW-37 [15], and UMI-77 [16]. These compounds vary in reported target specificity from pan-inhibition to MCL-1specific binding and have IC50 values in the upper nanomolar to micromolar range. Most of these compounds show little or no activity in iBH3 assays, indicating a lack of activity on mitochondria under conditions where there is no plasma membrane to serve as a barrier. However, all six compounds demonstrated an ability to kill primarily BFL-1- and MCL-1dependent cells (Fig. 2). This suggests that these compounds may be able to engage the BCL-2 family indirectly by influencing protein level or modifications upstream, but not directly at the mitochondrial level. Prior studies have shown that certain putative $\mathrm{BH} 3$ mimetics increase NOXA protein levels, leading indirectly to inhibition of MCL-1 and cell death [3, 17-20]. Obatoclax [3], and to a lesser degree gossypol, caused death in BAX/BAK DKO cells, indicating their ability to also kill independent of intrinsic apoptosis. At the mitochondrial level, obatoclax caused cytochrome $\mathrm{c}$ release in mitochondria lacking BAX and BAK at similar concentrations to the other cell lines, implying it may have a direct membrane lytic function at the mitochondria at high concentrations. All six compounds failed to show strong cytochrome $\mathrm{c}$ release in $\mathrm{iBH} 3$ despite being able to selectively kill cells, and so these compounds cannot be considered potent direct BH3 mimetics. We cannot rule out participation of these compounds in signaling events upstream of the mitochondrion that less directly induce apoptosis via the mitochondrial, or intrinsic, pathway.
A
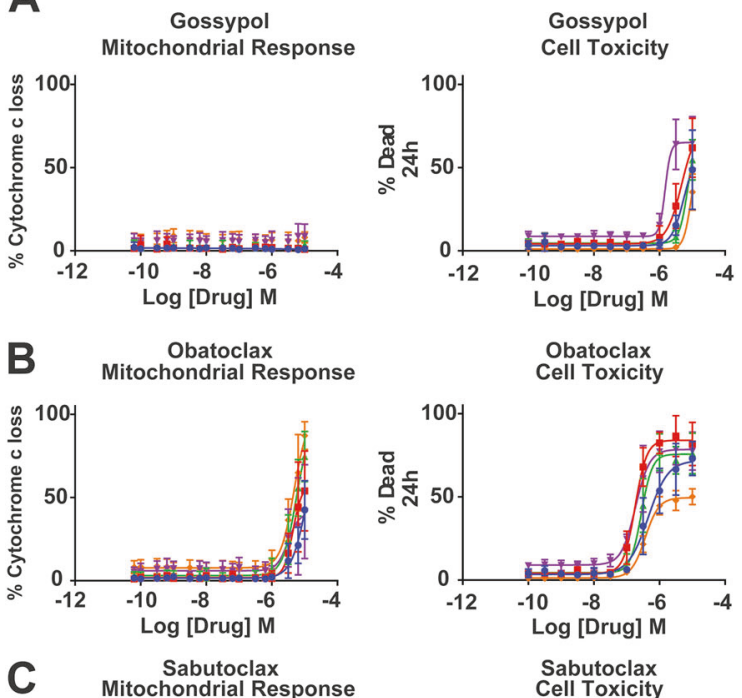

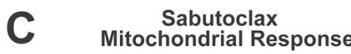
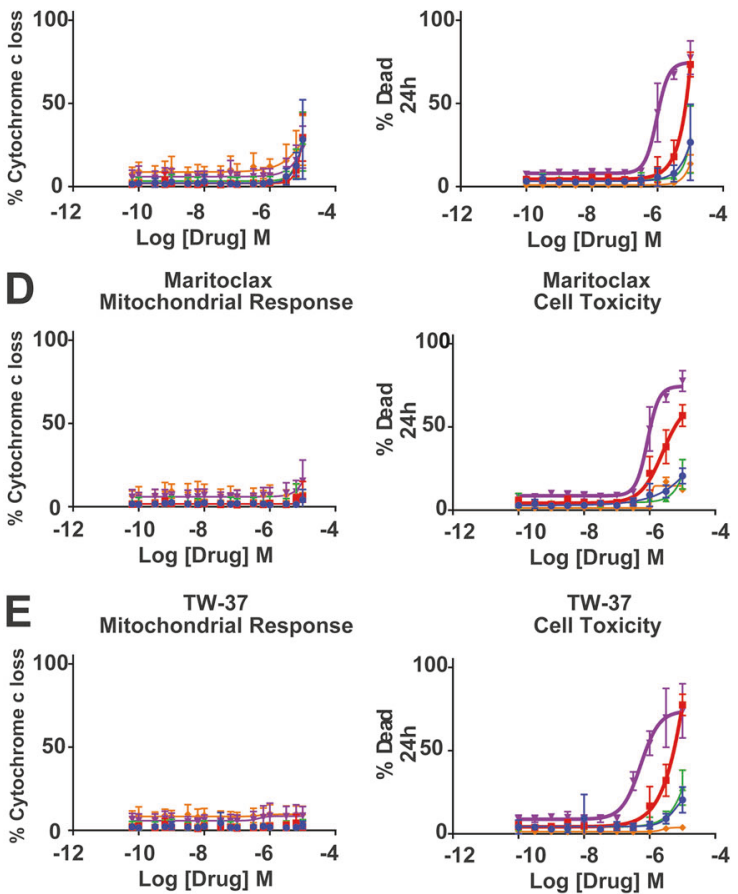

TW-37
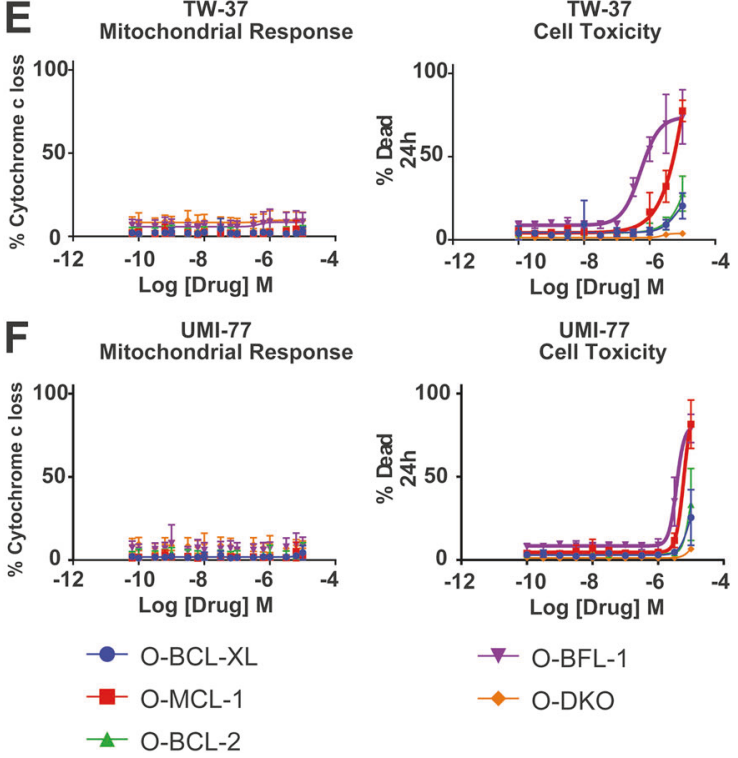

We next considered three available putative BCL-2/ BCL-XL dual inhibitors: ABT-737 [21], ABT-263 [22], and AZD4320 [23]. (Fig. 3) All three compounds caused 
Fig. 2 iBH3 and toxicity of putative BH3 mimetics. Direct mitochondrial response by $\mathrm{iBH} 3$ at $1 \mathrm{~h}$ (left) and cell killing as measured by Annexin $\mathrm{V}$ positivity at $24 \mathrm{~h}$ (right) using each of the five BCL-2 family model lines exposed to a gossypol, b obatoclax, $\mathbf{c}$ maritoclax, d sabutoclax, e TW37, and f UMI-77. For mitochondrial activity $N=6$ or more and for viability $N=3$ or more. Bars indicate standard deviation

A

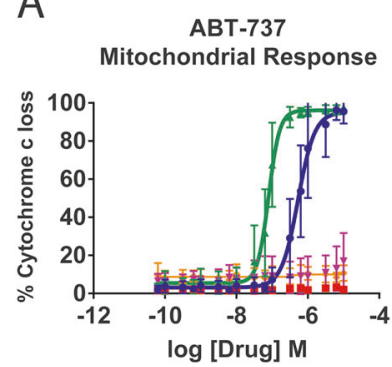

B

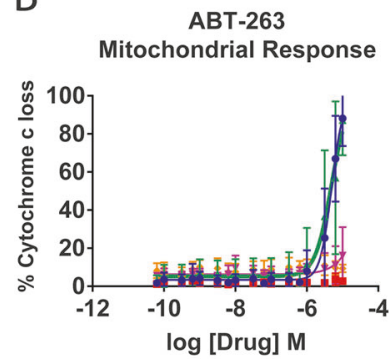

C
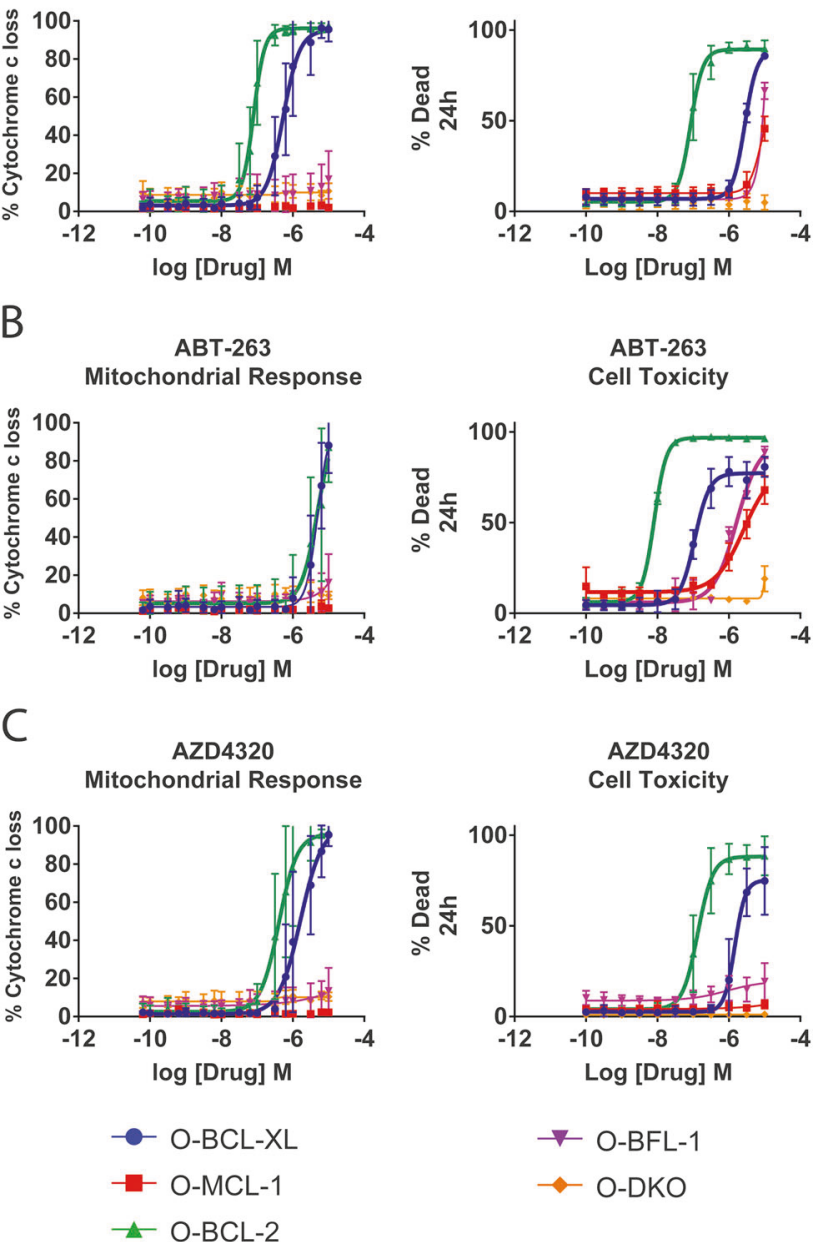

Fig. 3 Cell killing by dual BCL2/BCLXL inhibitors matches iBH3 direct mitochondrial response. Direct mitochondrial response by $\mathrm{iBH} 3$ at $1 \mathrm{~h}$ (left) and cell killing as measured by Annexin V positivity at $24 \mathrm{~h}$ (right). Mitochondria exposed to dual inhibitors release cytochrome $\mathrm{c}$ only if BCL-2- or BCL-XL-dependent. For mitochondrial activity $N=6$ or more and for viability $N=3$ or more. Bars indicate standard deviation

cytochrome $\mathrm{c}$ release from BCL-2- and BCL-XL-dependent mitochondria without a response from mitochondria of any other dependency. They also killed only those cells with the same dependencies as seen in the mitochondrial assay, although ABT-737 is relatively inefficient in killing the BCL-XL-dependent cells. We detected no response from the BAX/BAK DKO control from any compound, which is
A
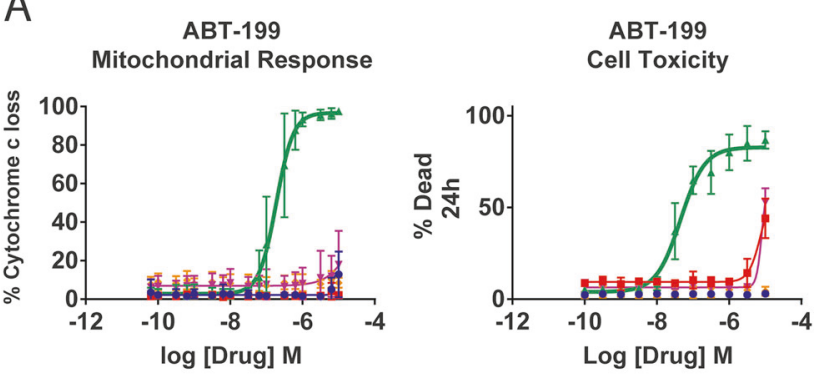

B
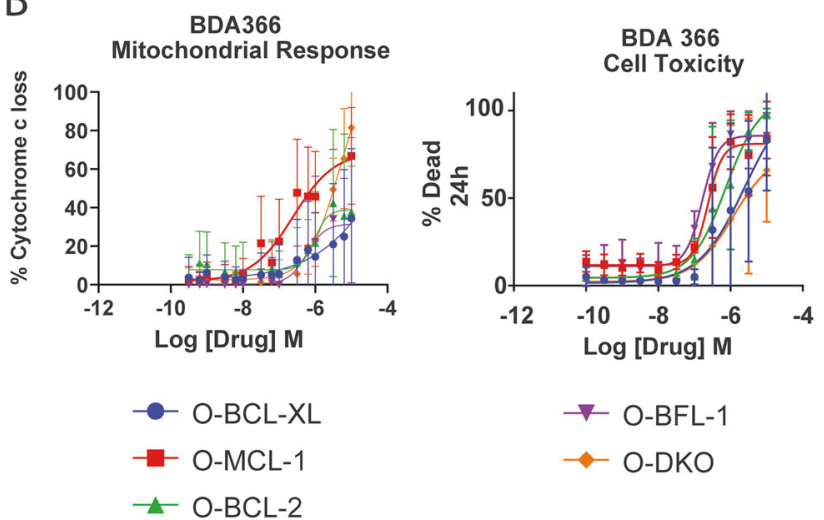

Fig. 4 a The BCL-2 specific inhibitor venetoclax (ABT-199) selectively causes cytochrome $\mathrm{c}$ release and cell killing if the cells are BCL-2-dependent. $\mathrm{iBH} 3$ response at $1 \mathrm{~h}$ and Annexin $\mathrm{V}$ positivity at $24 \mathrm{~h}$ is shown. b The putative BH4 BCL-2 antagonist lacks BCL-2 selective effect at the mitochondrial or cellular level. For mitochondrial activity $N=6$ or more and for viability $N=3$ or more. Bars indicate standard deviation

consistent with these compounds possessing on-target, mitochondrial-dependent killing only through competitive binding with their reported targets at the mitochondria. Similar observations of potent and selective cellular action of these compounds have also been reported [24].

The more recent $\mathrm{BH} 3$ mimetics focus on inhibiting a single anti-apoptotic protein. The effort is partly born out of a desire to avoid side effects. On-target thrombocytopenia in trials using ABT-263 inhibiting BCL-XL-dependent mature platelets lead to the synthesis of ABT-199 [10], a BCL-2 selective inhibitor that is now in clinical trials and approved by the FDA for CLL and AML. When tested with our toolkit, only BCL-2-dependent mitochondria responded, and only BCL-2-dependent cells were killed. In both iBH3 and Annexin V assays, ABT-199 showed activity in the low to mid-nanomolar range. Given the clinical success of this compound, future $\mathrm{BH} 3$ mimetics should be expected to perform to a similar degree if they are to be effective (Fig. 4a). We also studied compound BDA-366 [25, 26]. BDA-366 is reported to selectively inhibit BCL-2 protein function by binding to the BH4 domain of BCL-2. As such, it would also be expected to be selectively toxic to BCL-2dependent cells and mitochondria. The results in Fig. 4b 
A
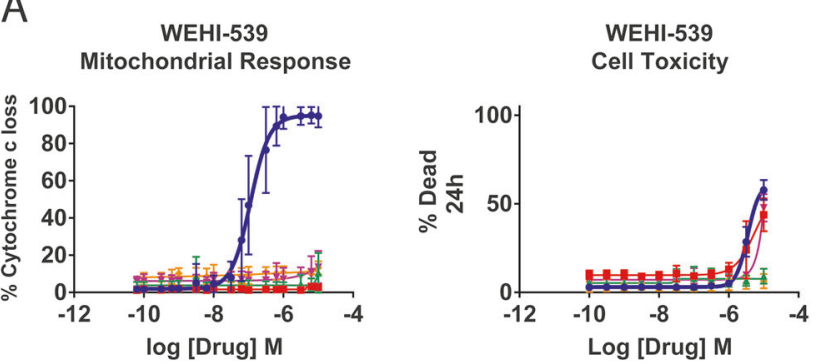

B

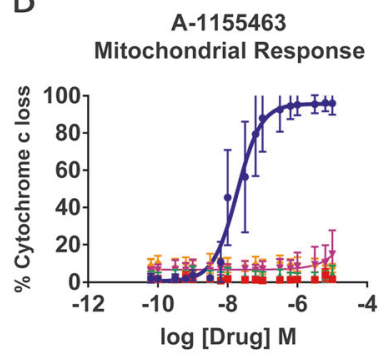

C
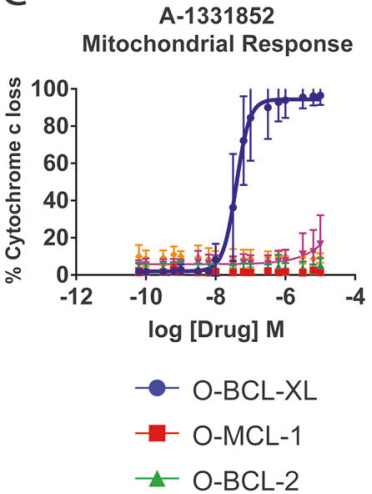

Fig. 5 The BCL-XL specific inhibitors a WEHI-539, b A-1155463, and c A-1331852 selectively cause cytochrome c release and cell killing if the cells are BCL-XL-dependent. iBH3 response at $1 \mathrm{~h}$ and Annexin $\mathrm{V}$ positivity at $24 \mathrm{~h}$ is shown. For mitochondrial activity $N=$ 6 or more and for viability $N=3$ or more. Bars indicate standard deviation

suggest that the mechanism of action of BDA-366 is not selective BCL-2 antagonism, as it lacks specificity for BCL2-dependent mitochondria or cells.

Three compounds, WEHI-539 [27], A-1155463 [28], and A-1331852 [29], are reported to have BCL-XL as a selective target. These three indeed inhibit BCL-XL alone, with all three showing strong, selective release of cytochrome $\mathrm{c}$ from mitochondria in the low nanomolar range (Fig. 5). The three compounds show different potencies in cellular Annexin $\mathrm{V}$ assays that may reflect serum binding or other issues involving cell entry and their ability to reach their target. Notably, WEHI-539 is relatively inefficient in killing BCL-XL-dependent cells. The most recent, A1331852 , is the most potent BCL-XL inhibitor by ${ }^{\mathrm{BBH}} 3$ and Annexin V. All three compounds show some off-target killing at $10 \mu \mathrm{M}$, but none of these compounds show toxicity or cytochrome $\mathrm{c}$ release in the BAX/BAK control cells. It may be possible that binding to other targets is occurring at high concentrations.

MCL-1 inhibitors that functioned by direct MCL-1 binding rather than by interfering with protein or transcript levels long remained elusive. However, four new compounds emerged recently, and so we applied our toolkit to see if they did indeed act in a mitochondria-dependent manner. A-1210477 [30], S63845 [31], AMG176 [32], and AZD5991 [33], listed in order of increasing potency in iBH3, caused cytochrome c release only from MCL-1dependent mitochondria (Fig. 6). BFL-1, the closest antiapoptotic to MCL-1 based on its ability to bind BH3-only proteins, does not respond at any dose of these compounds at the mitochondrial level. The order of potency is preserved in Annexin V cell death assays. A-1210477 is almost inert even at high doses even though it has activity at the mitochondria, suggesting that cell entry could be a barrier to its function. The remaining compounds are both specific at the cellular level even at high doses, with response from BFL-1 being the common off-target dependency.

\section{Discussion}

In this paper, we used a combination of mitochondrial and cellular assays of known dependence on individual antiapoptotic proteins to validate the mechanism of action of putative BH3 mimetics. For us to credential a compound as a true $\mathrm{BH} 3$ mimetic, it had to demonstrate the following:

(1) Selective induction of MOMP in mitochondria from cells of known dependence on the putative antiapoptotic protein target (e.g., a putative MCL-1 inhibitor should selectively induce MOMP in the mitochondria from the MCL-1-dependent cell line).

(2) Selective killing of cells of known dependence on the putative anti-apoptotic protein target (e.g., a putative MCL-1 inhibitor should kill the MCL-1-dependent cell line.)

(3) Lack of MOMP induction in mitochondria lacking BAX and BAK, and lack of killing of cell lines lacking BAX and BAK.

By these criteria, 11 of the tested compounds performed as true BH3 mimetics, including ABT-737, ABT-263, and AZD4320 (BCL-2 and BCL-XL inhibitors); ABT-199 (BCL-2 inhibitor); WEHI-539, A-1155463, and A1331852 (BCL-XL inhibitors); and A-1210477, S63845, AMG176, and AZD5991 (MCL-1 inhibitors). However, while each is a validated $\mathrm{BH} 3$ mimetic, this is not to say that all performed equivalently. For instance, among the BCL-2 
A

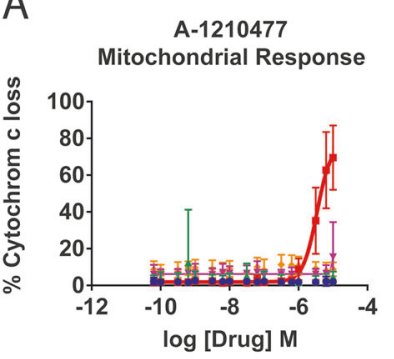

B

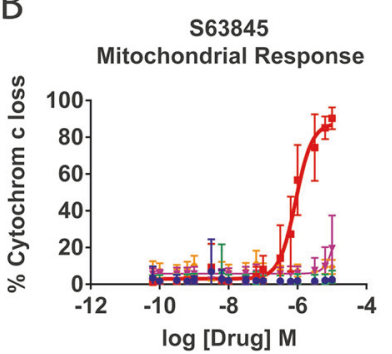

C

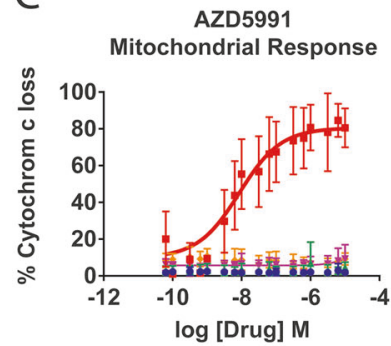

D

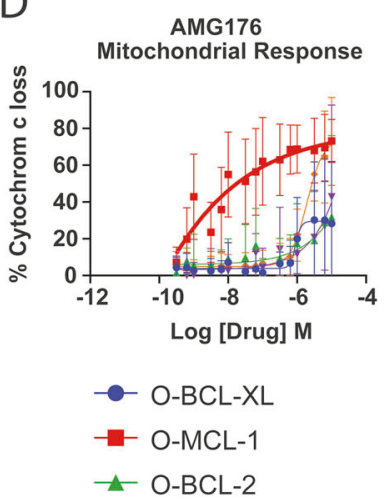

A-1210477

Cell Toxicity
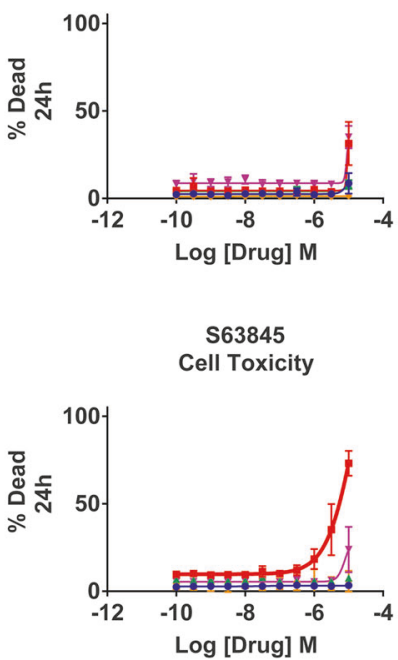

AZD5991

Cell Toxicity
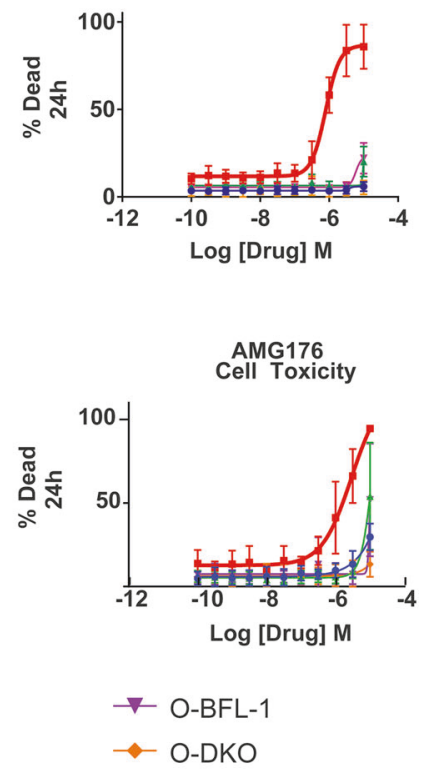

Fig. 6 The MCL-1 specific inhibitors a A1210477, b S63845, c AZD5991, and d AMG176 selectively cause cytochrome c release and cell killing if the cells are MCL-1 dependent. iBH3 response at $1 \mathrm{~h}$ and Annexin $\mathrm{V}$ positivity at $24 \mathrm{~h}$ is shown. For mitochondrial activity $N=6$ or more and for viability $N=3$ or more. Bars indicate standard deviation

and BCL-XL inhibitors, ABT-737 killed BCL-XLdependent cells less well than the other two, while ABT263 had less potency in mitochondrial assays. Among the selective BCL-XL inhibitors, WEHI-539 demonstrated less potent killing of the BCL-XL-dependent cell line. Among the selective MCL-1 inhibitors, A-1210477 showed inefficient killing of the MCL-1-dependent cell line. Moreover,

the extremely potent and selective induction of MOMP in MCL-1-dependent mitochondria by AZD5991 is notable, making it the most potent MCL-1 inhibitor at both the mitochondrial and cellular level of those we tested.

Seven of the test compounds did not perform as true BH3 mimetics and should be considered poor candidates for $\mathrm{BH} 3$ mimetic tool compounds or drugs. Gossypol, maritoclax, TW37, and UMI-77 demonstrated no mitochondrial activity at concentrations up to $10 \mu \mathrm{M}$. While sabutoclax, BDA-366, and especially obatoclax demonstrated mitochondrial activity at concentrations $>1 \mu \mathrm{M}$, it is notable that they were similarly active on all mitochondria, including those lacking BAX and BAK. This strongly suggests a mechanism of action independent of the BCL-2 family.

It is worth asking how compounds lacking on-target mechanism nonetheless advanced as putative $\mathrm{BH} 3$ mimetics. In each case, these drugs were found to bind at least one antiapoptotic BCL-2 family protein in a biochemical assay, although generally with modest $(>100 \mathrm{nM})$ affinity. Also, in each case, these drugs demonstrated the ability to kill cells. In some cases, killing of MCL-1-dependent cells was taken as evidence that the drug selectively targeted MCL-1 [12-14]. However, we have shown previously that many drugs that do not target MCL-1 directly nonetheless selectively target MCL-1-dependent cells, perhaps because of the relative short half-life of the MCL-1 protein [2]. What was lacking in prior studies was the demonstration that the killing was taking place via direct targeting of mitochondrial BCL-2 family proteins, an absolute requirement for a true $\mathrm{BH} 3$ mimetic. When finally subjected to mitochondrial testing (Fig. 2), these compounds failed to demonstrate the required activity. We suggest that to avoid similar confusion in the future, all putative BH3 mimetic compounds should be subjected to a direct mitochondrial assay using mitochondria of validated dependence on anti-apoptotic BCL-2 family proteins.

It is notable that other laboratories, using different methods, have identified a lack of on-target activity for six of the drugs studied here [34-37]. It is equally notable that, notwithstanding this information, with the exception of maritoclax, even through 2018 there are publications using these compounds as if they were on-target $\mathrm{BH} 3$ mimetics. There are now unambiguously potent and selective $\mathrm{BH} 3$ mimetic inhibitors of BCL-2, BCL-XL, and MCL-1, including those we have identified above. At least one of each specificity is available from at least one commercial vendor each. We strongly urge cell death investigators interested in $\mathrm{BH} 3$ mimetics to restrict their experimentation to compounds with a thoroughly validated mechanism of action, and to avoid those that clearly lack such a mechanism.

Acknowledgements We would like to thank AstraZeneca for providing both AZD4320 and AZD5991. We gratefully acknowledge 
funding support from the Ludwig Center at Harvard and NCI for AL R01CA205967 and JTO R01CA201069.

\section{Compliance with ethical standards}

Conflict of interest JR is a consultant for Vivid Bioscience. AL is a cofounder and equity holder in Flash Therapeutics and Vivid Bioscience. His laboratory has received research support from Novartis, AbbVie and Astra-Zeneca. JTO has received research support from AbbVie. AL has received consulting fees from AbbVie. The remaining authors declare that they have no conflict of interest.

Publisher's note: Springer Nature remains neutral with regard to jurisdictional claims in published maps and institutional affiliations.

\section{References}

1. Hanahan D, Weinberg RA. The hallmarks of cancer. Cell. 2000;100:57-70.

2. Brunelle JK, Ryan J, Yecies D, Opferman JT, Letai A. MCL-1dependent leukemia cells are more sensitive to chemotherapy than BCL-2-dependent counterparts. J Cell Biol. 2009;187:429-42.

3. Soderquist RS, Eastman A. BCL2 inhibitors as anticancer drugs: a plethora of misleading $\mathrm{BH} 3$ mimetics. Mol Cancer Ther. 2016;15:2011-7.

4. Koss B, Ryan J, Budhraja A, Szarama K, Yang X, Bathina M, et al. Defining specificity and on-target activity of BH3-mimetics using engineered B-ALL cell lines. Oncotarget. 2016;7:11500-11.

5. Certo M, Del Gaizo Moore V, Nishino M, Wei G, Korsmeyer S, Armstrong SA, et al. Mitochondria primed by death signals determine cellular addiction to antiapoptotic BCL-2 family members. Cancer cell. 2006;9:351-65.

6. Foight GW, Ryan JA, Gulla SV, Letai A, Keating AE. Designed BH3 peptides with high affinity and specificity for targeting Mcl-1 in cells. ACS Chem Biol. 2014;9:1962-8.

7. Jenson JM, Ryan JA, Grant RA, Letai A, Keating AE. Epistatic mutations in PUMA BH3 drive an alternate binding mode to potently and selectively inhibit anti-apoptotic Bfl-1. elife. 2017;6: e25541.

8. Ryan J, Montero J, Rocco J, Letai A. iBH3: simple, fixable BH3 profiling to determine apoptotic priming in primary tissue by flow cytometry. Biol Chem. 2016;397:671-8.

9. Brumatti G, Sheridan C, Martin SJ. Expression and purification of recombinant annexin $\mathrm{V}$ for the detection of membrane alterations on apoptotic cells. Methods. 2008;44:235-40.

10. Souers AJ, Leverson JD, Boghaert ER, Ackler SL, Catron ND, Chen J, et al. ABT-199, a potent and selective BCL-2 inhibitor, achieves antitumor activity while sparing platelets. Nat Med. 2013;19:202-8.

11. Balakrishnan K, Wierda WG, Keating MJ, Gandhi V. Gossypol, a BH3 mimetic, induces apoptosis in chronic lymphocytic leukemia cells. Blood. 2008;112:1971-80.

12. Nguyen M, Marcellus RC, Roulston A, Watson M, Serfass L, Murthy Madiraju SR, et al. Small molecule obatoclax (GX15-070) antagonizes MCL-1 and overcomes MCL-1-mediated resistance to apoptosis. Proc Natl Acad Sci USA. 2007;104:19512-7.

13. Dash R, Azab B, Quinn BA, Shen X, Wang XY, Das SK, et al. Apogossypol derivative BI-97C1 (Sabutoclax) targeting Mcl-1 sensitizes prostate cancer cells to mda-7/IL-24-mediated toxicity. Proc Natl Acad Sci USA. 2011;108:8785-90.

14. Doi K, Li R, Sung SS, Wu H, Liu Y, Manieri W, et al. Discovery of marinopyrrole A (maritoclax) as a selective Mcl-1 antagonist that overcomes ABT-737 resistance by binding to and targeting Mcl-1 for proteasomal degradation. J Biol Chem. 2012;287:10224-35.

15. Verhaegen M, Bauer JA, Martin de la Vega C, Wang G, Wolter $\mathrm{KG}$, Brenner JC, et al. A novel BH3 mimetic reveals a mitogenactivated protein kinase-dependent mechanism of melanoma cell death controlled by p53 and reactive oxygen species. Cancer Res. 2006;66:11348-59.

16. Abulwerdi F, Liao C, Liu M, Azmi AS, Aboukameel A, Mady AS, et al. A novel small-molecule inhibitor of mcl-1 blocks pancreatic cancer growth in vitro and in vivo. Mol Cancer Ther. 2014;13:565-75.

17. Albershardt TC, Salerni BL, Soderquist RS, Bates DJ, Pletnev AA, Kisselev AF, et al. Multiple BH3 mimetics antagonize antiapoptotic MCL1 protein by inducing the endoplasmic reticulum stress response and up-regulating BH3-only protein NOXA. J Biol Chem. 2011;286:24882-95.

18. Mallick DJ, Soderquist RS, Bates D, Eastman A. Confounding offtarget effects of BH3 mimetics at commonly used concentrations: MIM1, UMI-77, and A-1210477. Cell Death Dis. 2019;10:185.

19. Soderquist R, Pletnev AA, Danilov AV, Eastman A. The putative BH3 mimetic S1 sensitizes leukemia to ABT-737 by increasing reactive oxygen species, inducing endoplasmic reticulum stress, and upregulating the $\mathrm{BH} 3$-only protein NOXA. Apoptosis. 2014;19:201-9.

20. Soderquist RS, Danilov AV, Eastman A. Gossypol increases expression of the pro-apoptotic BH3-only protein NOXA through a novel mechanism involving phospholipase A2, cytoplasmic calcium, and endoplasmic reticulum stress. J Biol Chem. 2014;289:16190-9.

21. Oltersdorf T, Elmore SW, Shoemaker AR, Armstrong RC, Augeri DJ, Belli BA, et al. An inhibitor of Bcl-2 family proteins induces regression of solid tumours. Nature. 2005;435:677-81.

22. Tse C, Shoemaker AR, Adickes J, Anderson MG, Chen J, Jin S, et al. ABT-263: a potent and orally bioavailable Bcl-2 family inhibitor. Cancer Res. 2008;68:3421-8.

23. Cidado J, Secrist JP, Gibbons FD, Hennessy EJ, Ioannidis S, Clark EA. Abstract 311: AZD4320 is a potent, dual Bcl-2/xLinhibitor that rapidly induces apoptosis in preclinical hematologic tumor models. Cancer Res. 2018;78:311.

24. Lopez J, Bessou M, Riley JS, Giampazolias E, Todt F, Rochegue $\mathrm{T}$, et al. Mito-priming as a method to engineer Bcl-2 addiction. Nat Commun. 2016;7:10538.

25. Deng J, Park D, Wang M, Nooka A, Deng Q, Matulis S, et al. BCL2-BH4 antagonist BDA-366 suppresses human myeloma growth. Oncotarget. 2016;7:27753-63.

26. Han B, Park D, Li R, Xie M, Owonikoko TK, Zhang G, et al. Small-molecule Bcl2 $\mathrm{BH} 4$ antagonist for lung cancer therapy. Cancer cell. 2015;27:852-63.

27. Lessene G, Czabotar PE, Sleebs BE, Zobel K, Lowes KN, Adams JM, et al. Structure-guided design of a selective BCL-X(L) inhibitor. Nat Chem Biol. 2013;9:390-7.

28. Tao ZF, Hasvold L, Wang L, Wang X, Petros AM, Park CH, et al. Discovery of a potent and selective BCL-XL inhibitor with in vivo activity. ACS Med Chem Lett. 2014;5:1088-93.

29. Leverson JD, Phillips DC, Mitten MJ, Boghaert ER, Diaz D, Tahir SK, et al. Exploiting selective BCL-2 family inhibitors to dissect cell survival dependencies and define improved strategies for cancer therapy. Sci Transl Med. 2015;7:279ra40.

30. Leverson JD, Zhang H, Chen J, Tahir SK, Phillips DC, Xue J, et al. Potent and selective small-molecule MCL-1 inhibitors demonstrate on-target cancer cell killing activity as single agents and in combination with ABT-263 (navitoclax). Cell Death Dis. 2015;6:e1590.

31. Kotschy A, Szlavik Z, Murray J, Davidson J, Maragno AL, Le Toumelin-Braizat G, et al. The MCL1 inhibitor S63845 is 
tolerable and effective in diverse cancer models. Nature. 2016;538:477-82.

32. Caenepeel S, Brown SP, Belmontes B, Moody G, Keegan KS, Chui D, et al. AMG 176, a selective MCL1 inhibitor, is effective in hematologic cancer models alone and in combination with established therapies. Cancer Disco. 2018;8:1582-97.

33. Tron A, Belmonte M, Adam A, Aquila B, Boise L, Chiarparin E, et al. Discovery of Mcl-1-specific inhibitor AZD5991 and preclinical activity in multiple myeloma and acute myeloid leukemia. Nat Commun. 2018;9:5341.

34. Varadarajan S, Poornima P, Milani M, Gowda K, Amin S, Wang $\mathrm{HG}$, et al. Maritoclax and dinaciclib inhibit MCL-1 activity and induce apoptosis in both a MCL-1-dependent and -independent manner. Oncotarget. 2015;6:12668-81.

35. Varadarajan S, Vogler M, Butterworth M, Dinsdale D, Walensky LD, Cohen GM. Evaluation and critical assessment of putative MCL-1 inhibitors. Cell Death Differ. 2013;20:1475-84.

36. Vogler M, Weber K, Dinsdale D, Schmitz I, Schulze-Osthoff K, Dyer MJ, et al. Different forms of cell death induced by putative BCL2 inhibitors. Cell Death Differ. 2009;16:1030-9.

37. Varadarajan S, Butterworth M, Wei J, Pellecchia M, Dinsdale D, Cohen GM. Sabutoclax (BI97C1) and BI112D1, putative inhibitors of MCL-1, induce mitochondrial fragmentation either upstream of or independent of apoptosis. Neoplasia. 2013;15:568-78. 